Proc. Indian Acad. Sci. (Earth Planet, Sci.), Vol. 90, Number 1, March 1981, pp. 75-83. (C) Printed in India.

\title{
A T-shaped four-electrode configuration for resistivity profiling over thin vertical conducting veins
}

\author{
S V SURYANARAYANA RAO, S MURALI and \\ V L S BHIMASANKARAM \\ Centro of Bxploration Geophysies, Department of Geophysics, Osmania University \\ Hydorabad 500 007, India
}

MS received 29 June 1979; revised 18 December 1980

\begin{abstract}
A new variant of the four-electrode system is proposed for geophysical resistivity investigations, particularly for profiling over conductive bodies. Model tank resistivity profiling experiments with Woaner, Schlumberger and the proposed T-shaped fcur-electrode arrays were carried out over a thin conducting vein type model to evaluate the efficacy of the new array. The proposed array appears to possess a larger response, a greater dopth of investigation and a higher vertical resolution in detacting conducting bodies, as compared to tho two-conventional arrays.
\end{abstract}

Keywords. Array; depth of investigation; depth of penetration; vertical resolution; four-electrode configuration; resistivity profile.

\section{Intradaction}

A large number of electrode arrays for electrical resistivity prospecting have been proposed during the last half a century. However apart from dipole-dipole arrays, most of the others are conventional four-electrode Wenner or Schlumberger arrays or their variants (Carpenter and Habberjam 1956; Sarma 1961; Zohdy 1970a, b). Several of the four-electrode arrays widely in use are colinear and symmetrical (Bhattacharya and Patra 1968) even though in recent times certain asymmetrical (Kunetz 1966; Heiland 1968) arrangements like the tri-electrode, two-electrode and uni-pole arrays have come into vogue. In this paper, a simple four-electrode, asymmetrical array is examined and is shown to possess some advantages over the two-symmetrical conventional arrays of Wenner and Schlumberger in locating conductive dyke-like bodies. By virtue of its shape, this array is called the 'T-shaped' four-electrode array.

\section{The array and its geomeric factor}

The proposed T-shaped array came to be examined as a result of the follawing considerations ;

(i) The conventional symmetrical electrode arrays produce an anomaly curve that is quite complicated even over a single anomalous body (depending on the 
ratios of its width and depth to the electrode spacing). Conductive bodies are even more difficult to be located by these symmetrical arrays.

(ii) While the uni-pole (Gupta and Bhattacharya 1963) or two-electrode (Roy and Appa Rao 1970) arrays may yield anomaly curves with simpler shapes, it is necessary to retain a certain degree of control on the depth of investigation by restricting the depth of penetration of current, which can be achieved only by using two or more source electrodes (Roy and Appa Rao 1971). In addition, two-source electrode arrays produce more or less uniform and horizontal electrical field near the centre of the electrodes which is preferable from many considerations, especially for profiling applications.

Thus it was felt necessary to experiment with a new electrode array which is asymmetric, has two source electrodes and is easy to work with under field conditions. The T-shaped four-electrode array is one of the many solutions that occurs readily to mind.

Essentially the proposed T-shaped four-electrode array consists of two source electrodes ( $A$ and $B$ ) and a measuring electrode $(M)$ arranged colinearly. The other measuring electrode $(N)$ is located on a perpendicular line intersecting $A B$ at $M$ (figure 1 ).

The geometric factor for this array is derived from the general expression

$$
R=2 \pi /(1 / A M-1 / B M-1 / A N+1 / B N),
$$

and is obtained as

$$
K_{\mathrm{r}}=2 \pi /\left[1 / A M-1 / B M-1 /\left(M N^{2}+A M^{2}\right)^{1 / 2}+1 /\left(M N^{2}+B N^{2}\right)^{1 / 2}\right]
$$

In the present investigations, the T-shaped four-electrode array with $A B=$ $3 A M=M N$ was used and in this case, the configuratin factor reduces to

$$
k_{\mathrm{x}}=4 \cdot 5 \mathrm{AB} \text {. }
$$

\section{Model tank experiments and results}

Modelling experiments by the profiling using the T-shaped four-electrode, Wenner and Schlumberger arrays were conducted in a model tank of length $3 \mathrm{~m}$, breadth $1.9 \mathrm{~m}$ and depth $1 \mathrm{~m}$ filled with tap water of resistivity of about $29 \mathrm{ohmm}$. All the experiments were conducted over the central region of the tank where wall effects are negligible.

The model vein was represented by an aluminium sheet of dimensions, $0.6 \mathrm{~m}$ $\times 0.5 \mathrm{~m} \times 0.012 \mathrm{~m}$. Obiervations were made with the above three arrays for different depths $(3,5,7,10,12$ and $15 \mathrm{~cm})$ of the model vein when it was vertical and striking normal to the profiles. All the observations were made at station intervals varying from $10 \mathrm{~cm}$ to $1 \mathrm{~cm}$ from a distance of about $100 \mathrm{~cm}$ on either side of the model vein.

The apparent resistivity measurements were carried out emplaying a low frequency resistivity equipment (LFR-1 made in USSR). In this equipment, a constant current generator provides $10 \mathrm{~mA}$ at $22.5 \mathrm{~Hz}$ for energisation, while the receiver is capable of measuring voltages from $10 \mu \mathrm{V}$ to $100 \mathrm{mV}$. The measuring unil reads $\Delta U / I$ values directly. 


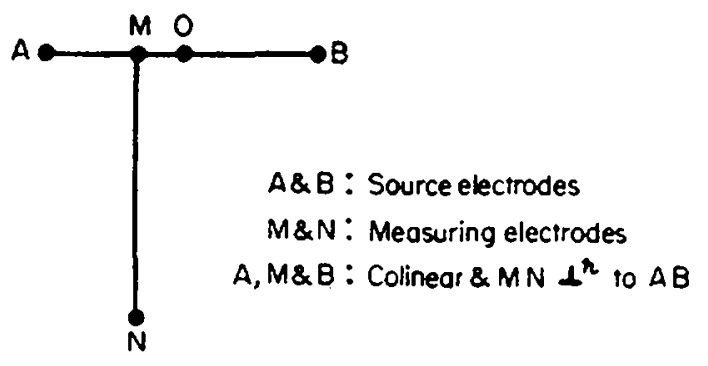

Flevere 1. The T-shaped four-aloctrode array.

With the above experimental set-up, observations were recorded for the following constant electrode separations for profiling :

T-shaped array : $\quad A B=M N=21 \mathrm{~cm} ; A M=7 \mathrm{~cm}$,

Wenper array: $\quad A B=21 \mathrm{~cm} ; M N=7 \mathrm{~cm}$,

Schlumberger array : $A B=21 \mathrm{~cm} ; M N=4 \mathrm{~cm}$.

Such separapions were chosen to facilitate comparison of results, as the current electrode separation in all the cases is the same which could provide an equal current distribution in depth for all the three arrays. Thus any variations in anomaly curves may be attributed to the inherent characteristics of the given array.

The results of profiling are represented as graphs of apparent resistivity along the profile against the locations of the configuration as referred to the mid-point ' $O$ ' of $A B$ for all the three arrays. The reforence point, in the present T-shaped asymmetrical system, is being defined as the "mid-point of the line between the two paired electrodes, which also accommodates one of the non-paired electrodes ".

The profiling curves obtained with the T-shaped array resemble in shape those obtained with the uni-pole and three electrode-AMN arrays, though in the present case the curve has an unequal peak and trough.

The results shown in figure 2 were obtained using the three arrays for the same depth of the body while it strikes normal to the profile and is vertical. Figures 3a, b, c show the results of profiling with T-shaped, Schlumberger and Wenner arrays, respectively over a vein located at varying depths.

The results abtained with model experiments lend themselves to the following analysis :

(i) The shape of the profiling curves in case of Wenner and Schlumberger arrays is similar, with two troughs flanking the body and a central peak over the body. In the T-shaped array, the two minima of the symmetrical arrays are converted into a peak and a low at corresponding locations. The profile for the T-shaped array is simpler and lends itself to easy recognition of the anomaly.

It may also be noted in passing that a conductive vein may produce in the symmetrical systems, an anomaly that changes its shape for increasing depths of the vein, from a ' $W$ 'shaped one at shallow depths to a broad ' $U$ ' at higher 


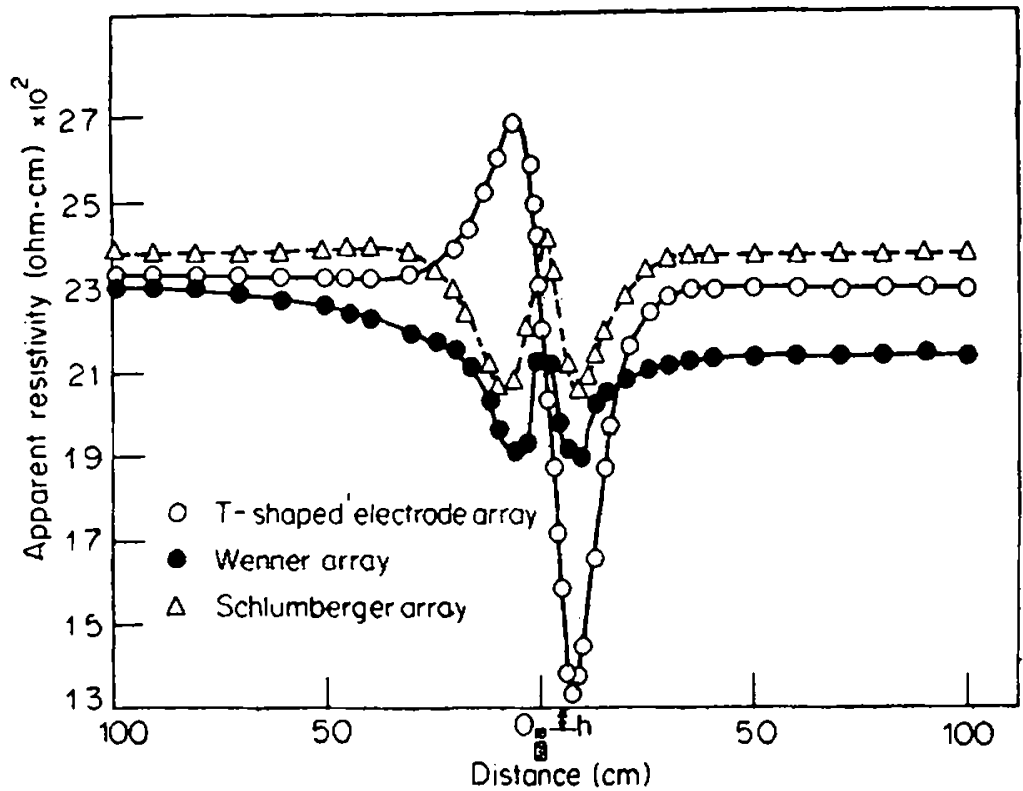

Figure 2. Resistivity profiles for $T$-shaped four-electrode, Wenner and Schlumberger configurations.

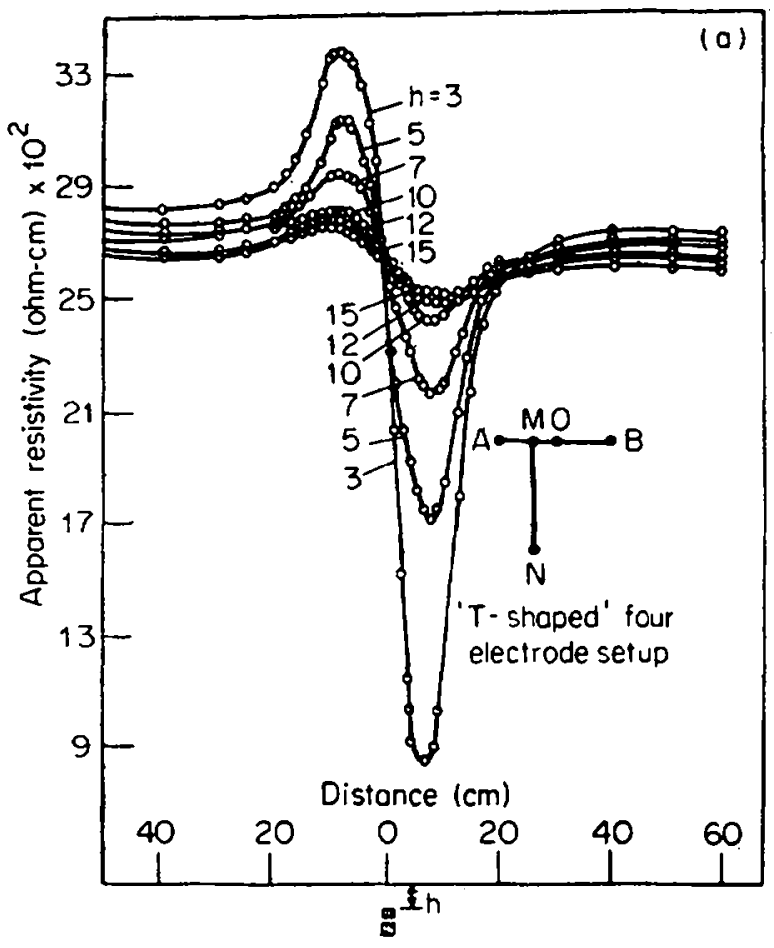

Figure 3. Resistivity profiles over a conducting vertical vetn at varying depths (index of curvo indicates depth in cm). a. T-shaped four electrodo b. Schlumberger. c. Weanes arrays. 


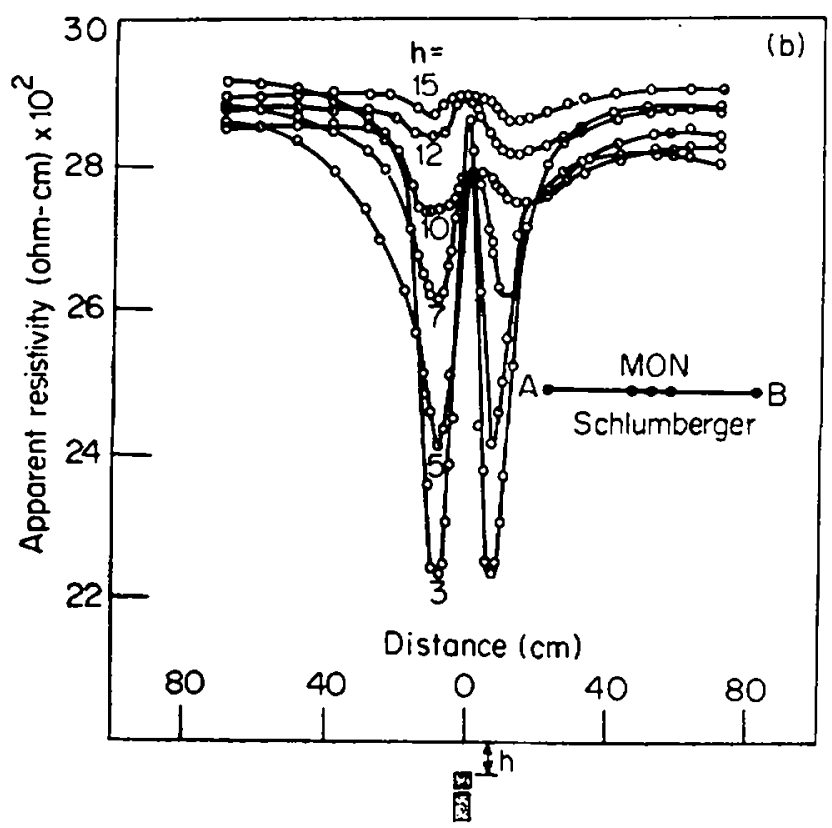

Fitgure 3b. Sec caption in p. 78 .

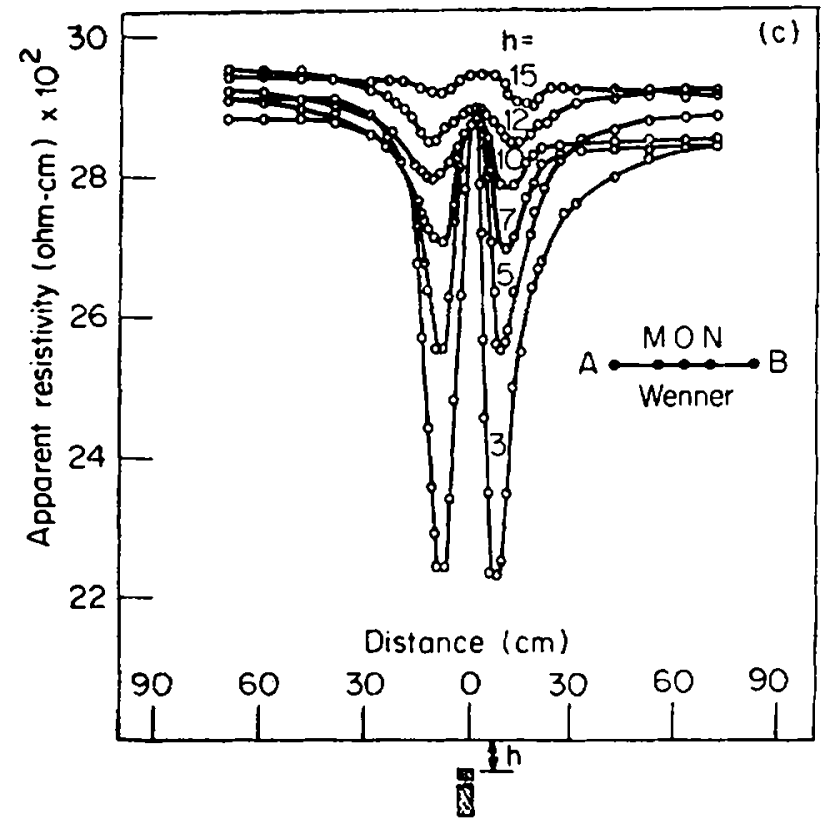

Figure 3e. See caption in $\mathbf{p}, 78$.

depths. The T-shaped array on the other hand yields only a single shape of the anomaly for all depths of body investigated. 
(ii) Besides the characteristic shape, the magnitude of the anomaly is higher with the T-shaped array as compared to the other two. As can be seen from figure 3, the magnitude of the anomaly for all depths of the model body (viz., 3 to $10 \mathrm{~cm}$ ) is about 3 times that of the Wenner anomaly and 2.5 times that of the Schlumberger anomaly. When the two symmetrical arrays failed to give any notable anomaly beyond the depth of $10 \mathrm{~cm}$, the $T$-shaped system could respond even upto $15 \mathrm{~cm}$ of the depth of the body.

(iii) Further, for providing a quantitative comparison, two characteristics of the arrays were examined. These are : (a) The depth of investigation and (b) (b) Vertical resolution, which may be defined as follows:

A given array may be said to possess a higher depth of investigation than another when it can detect the same anomalous body located at a greater depth. Obviously, this implies that whichever array yields the higher relative anomaly for a given body at a given depth has also a larger depth of investigation (or depth range). Since detection of the presence of an anomaly is dependent not only on the array, but also on operating conditions, equipment, etc., it is understood that these factors remain unchanged when the various arrays are compared.

The relative anomaly at any point for defining the depth of investigation was computed for the three arrays as follows:

$$
\rho_{\text {rol }}=\left(\rho_{\text {beckground }} \sim\left|\rho_{\mathrm{B}}\right|_{\text {Deak }}\right) / \rho_{\text {beckeround }}
$$

where $\rho_{\text {background, }}$ represents the smooth horizontal part of the profiling curve corresponding to the resistivity of water in the model tank. The computed values for the relative anomaly for the three arrays are shown in figure 4 plotted against the depth of the body. From this figure it may be seen that the relative anomaly with the T-shaped array is quite high compared to that of the other two arrays. For example at a body depth of $3 \mathrm{~cm}$, the Wenner and Schlumberger arrays show

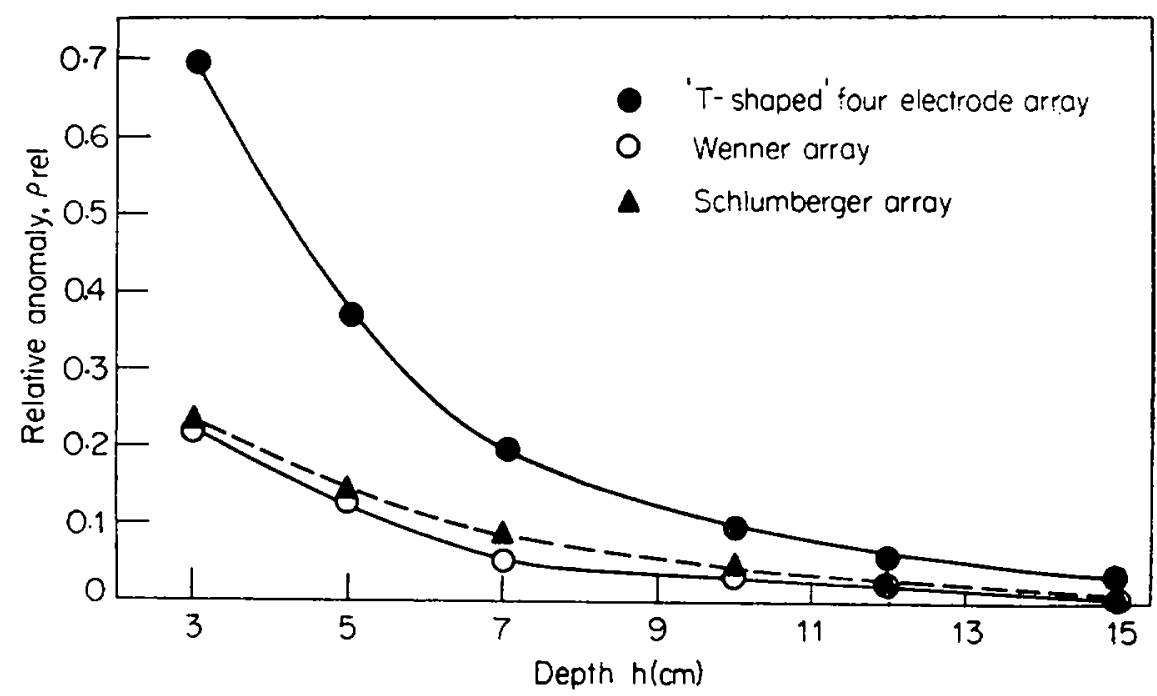

Figure 4. Comparison of dopth of investigation of various electrode arrays, 
a relative anomaly of about 0.20 while for the T-shaped array, it is about 0.6 . Likewise at a higher depth of $10 \mathrm{~cm}$, the symmetrical arrays indicate a relative anomaly of 0.04 , while for the T-shaped array, the anomaly is approximately 0.1 . It follows therefore that the relative anomaly and hence the depth of investigation is more in the T-shaped array at all depths compared to those of the other two arrays.

The vertical resolution of an electrode array may be defined as the change in relative anomaly $\left(d \rho_{\text {rei }}\right)$ for a given change in depth ( $\left.d h\right)$ of the same causative body. The vertical resolution for the various arrays is plotted against the depth of the body in figure 5. It is clear from the figure that the vertical resolution is higher in the T-shaped array than for the other two arrays by 3-4 times at the depth (less than $h=13 \mathrm{~cm}$ ) investigated. Also the symmetrical systems show a constant resclution at shallow depths and at depths exceeding 0.4 times $A B$, the resolution decreases. The T-shaped array on the other hand displays a large, though decreasing, resolution at depths upto 0.4 times $A B$, while for larger depths the resolution does not appear to decrease substantially. It may be noted that the resolution above depths of $9 \mathrm{~cm}$ is constant for Wenner and Schlumberger arrays, whereas it is more or less uniform above depths of $13 \mathrm{~cm}$ in the T-shaped array.

Among the two-symmetrical arrays, i.e., Wenner and Schlumberger, it can be seen that the Schlumberger always yields generally a higher relative anomaly and may be more convenient to work with as reported earlier (Bhimasankaram et al 1969).

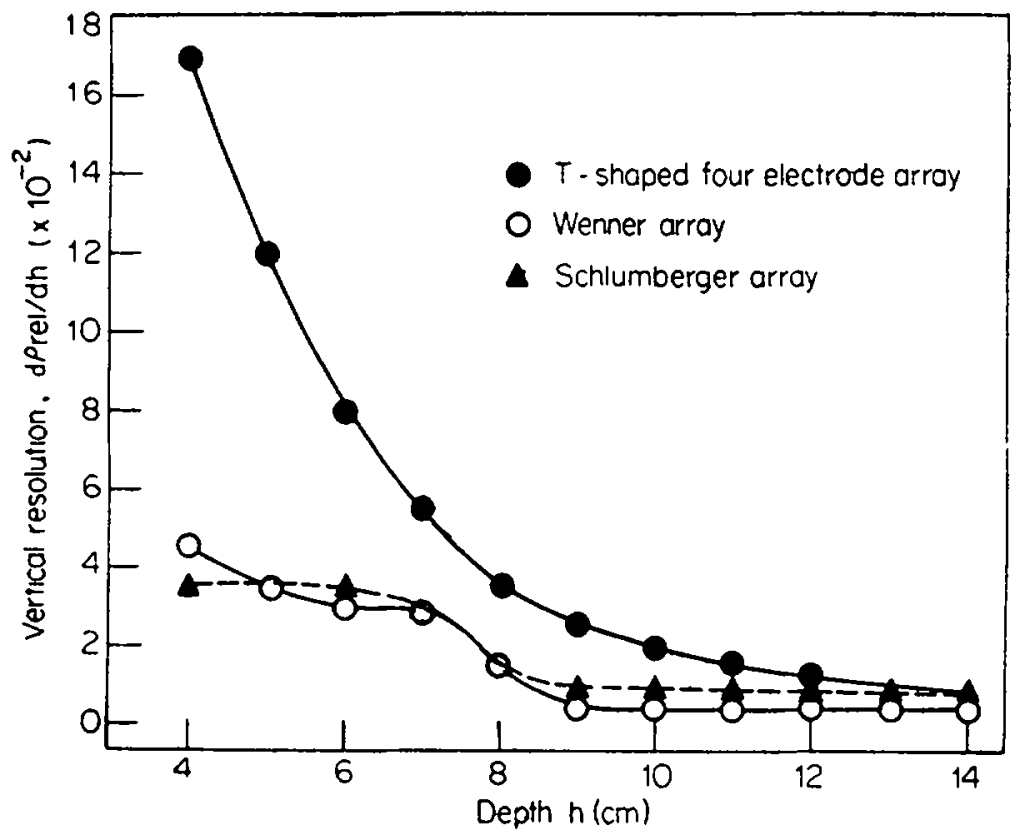

Figure 5. Comparison of vertical rosolution of various olectrode arrays. 


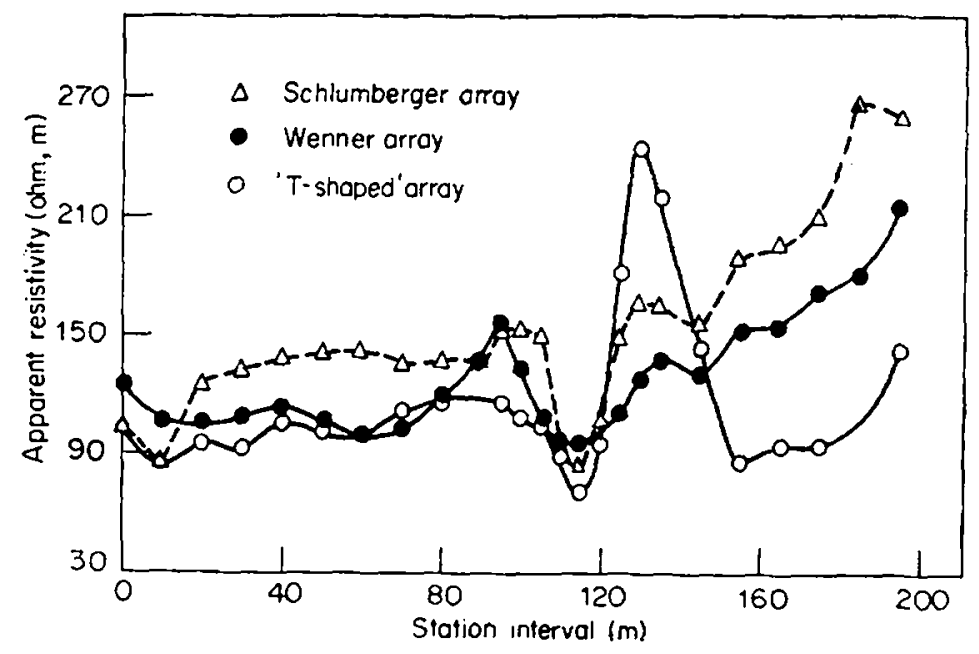

Flgure 6. Apparent resistivity field test profiles for T-shaped four-oloctrode, Wenner and Schlumberger arrays over a conductive dyke.

\section{Field application}

The T-shaped array was employed for resistivity profiling vis-a-vis the Wenner and Schlumberger arrays. The area has been described by Patangay (1977). The surveyed area is a typical granitic terrain and is fringed by exposures on all sides, while a dolerite dyke of $10-15 \mathrm{~m}$ thickness crosses roughly in east-west direction. The resistivity profiling was carried out in the north-south direction, i.e., approximately perpendicular to the strike of the dyke. For all the three arrays used namely, Wenner, Schlumberger and T-shaped, the current electrode separation $(A B)$ of $60 \mathrm{~m}$ was employed with corresponding potential electrode separations $(M N)$, i.e., $=20 \mathrm{~m}, 4 \mathrm{~m}$ and $60 \mathrm{~m}$ for Wenner, Schlumberger and T-shaped arrays respectively. The profile length of $200 \mathrm{~m}$ was covered by observations at an interval of $10 \mathrm{~m}$ reducing the intervals to $5 \mathrm{~m}$ over the dyke. The results are shown in figure 6, with apparent resistivity plotted for each station along the profile.

It will be seen that all the three arrays indicate a constant background value over the country rock between the stations, $0-80 \mathrm{~m}$ on the profile as 90 to $140 \mathrm{ohm}$ $\mathrm{m}$. The presence of a conductive dyke is clearly indicated by a ' $U$ '-shaped low between the points 108 and 130 by the Schlumberger array, whereas the Wenner array displays a slightly broader low corresponding to the almost same locations. The dyke is demarcated in a very distinctive fashion by the T-shaped array and the centre of the dyke may be located at $115 \mathrm{~cm}$, which also agrees with the location from the two other arrays. The apparent resistivity value measured over the dyke for the T-shaped array is much lower than for the other two arrays, thus supporting the results of modelling investigation. 


\section{Canclusions}

It may be stated that the new T-shaped array appears to possess a larger response, a greater depth of investigation and a higher vertical resolution in detecting conducting bodies as compared to conventional symmetrical systems. Also it has the advantage of a simpler and easily identifiable anomaly and possesses a controlable depth of penetration of current. By virtue of these qualities, the T-shaped array may be recommended in resistivity profiling applications for the location of conductive bodies.

\section{Ackrowledgements}

The authors thank the University Grants Commission for their aid in conducting the above work as also to Messis K K Sharma, H V Srinivas, M V Chary, Chandra Reddy, Shyam Sunder and Ikramullah for assistance.

\section{References}

Bhattacharya P K and Patra H P 1968 Direct current electric sounding (Amsterdam: Elsevier) Bhimasankaram V L S, Murali S and Tarkov A G 1969 Bull. Natl. Geophys. Res. Inst. 7159 Carpenter E W and Habberjam G M 1956 Geophysics 21455

Gupta R N and Bhattacharya P K 1963 Geophysics 28608

Heiland C A 1968 Geophysical exploration (New York and London: Hafner Publ. Co.)

Kunetz G 1966 Principles of DC resistivity prospecting, Geoexploration Monogr. (Berlin: GeoPublication Associates)

Patangay N S 1977 In Lectures on exploration geophysics for geologists and engineers (Hyderabad : AEG Publication)

Roy A and Appa Rao A 1970 Geo-exploration 9195

Roy A and Appa Rao A 1971 Geophysics 36943

Sarma V V J 1961 Geophys. Prospect. 9568

Zohdy Adel A R 1970a USGS Bull. B1313 26

Zohdy Adel A R 1970b USGS Bull. C1313 20. 